\title{
Incomplete Immune Recovery in HIV Infected Individuals After Long-Term Antiretroviral Therapy and The Role of Traditional Chinese Medicine
}

\author{
Zou Wen ${ }^{1,2}$, Wang Jian ${ }^{1}$, Liu Ying*1, Gao Guojian ${ }^{1}$, Dong Jipeng ${ }^{1}$ and Zhang Wei $^{3}$ \\ ${ }^{1}$ Center of AIDS Treatment with Traditional Chinese Medicine, China Academy of Chinese Medical Sciences,China \\ ${ }^{2}$ Institute of Basic Theory of Traditional Chinese Medicine, China Academy of Chinese Medical Sciences,China
}

${ }^{3}$ Beijing Ditan Hospital Capital Medical University, China

*Corresponding author: LIU Ying, Center of AIDS Treatment with Traditional Chinese Medicine, China Academy of Chinese Medical

Sciences, Beijing (100700), China

\section{ARTICLE INFO}

Received: 幽 October 24, 2019

Published: 慧 November 01, 2019

Citation: Zou Wen, Wang Jian1, Liu Ying, Gao Guojian, Dong Jipeng, Zhang Wei. Incomplete Immune Recovery in HIV Infected Individuals After Long-Term Antiretroviral Therapy and The Role of Traditional Chinese Medicine. Biomed J Sci \& Tech Res 22(3)-2019. BJSTR. MS.ID.003754.

\section{ABSTRACT}

Although treatment of HIV-infected patients with highly active antiretroviral therapy (HAART) causes a rapid reduction in HIV-RNA viral load and restoration of CD4+ T-cell numbers for most patients, up to $40 \%$ of individuals receiving HAART have incomplete immune recovery despite sustained HIV-RNA viral suppression. The reason for immunological nonresponse is incompletely understood. This paper summarizes the main factors regarding the incomplete immune reconstitution in HIV infection and the role of traditional Chinese medicine to improve immune reconstitution.

Keywords: Incomplete Immune Recovery; HIV Immune Activation; Traditional Chinese Medicine

\section{Introduction}

Human immunodeficiency virus (HIV) care continuum attrition is a major global public health challenge [1]. Treatment of HIVinfected patients with highly active antiretroviral therapy (HAART) usually results in diminished viral replication, increasing CD4+T cell counts, a reversal of most immunological disturbances, and a reduction in risk of morbidity and mortality. Although HAART causes a rapid reduction in HIV-RNA viral load and restoration of CD4+ T-cell numbers for most patients, up to $30 \%$ of individuals receiving HAART have incomplete immune recovery despite sustained HIV-RNA viral suppression [2-4]. Most often incomplete immune recovery is defined as having CD4+ T-cell counts $<200$ cells $/ \mu \mathrm{L}$ or $\mathrm{CD} 4+\mathrm{T}$-cell increase in percentages $<20 \%$ increase from baseline [5-6]. These individuals with CD4+ T-cell counts $<200$ cells $/ \mu \mathrm{L}$ are referred to as immunological non responders (INRs). Patients with CD4+ T-cell counts between 200 and 500 cells/ $\mu \mathrm{L}$ are consequently classified as inadequate immune responders (IIR) which may have a morbidity and mortality rate distinct from INR as well as from those with adequate immune response [7]. Patients with an adequate immune response to HAART including those with a CD4+ T-cell count $>500$ cells $/ \mu \mathrm{L}$ often have a morbidity and mortality rate comparable to those of HIV negative individuals. It is widely accepted that the increased risks of long-term morbidity and mortality correlated with status of INRs or IIR [8-9].

\section{The Mechanism of Poor Immune Reconstitution}

There is a general consensus that $\mathrm{T}$ cell reconstitution is a three-phase process, the first fast phase occurs during the first 6 months and is mostly due to memory $\mathrm{T}$ cell redistribution. The last two slower phases takes several additional years [10-11]. The mechanism of poor immune reconstitution may depend on several factors including older age, a long duration of the HIV infection prior to HAART, co-infection with hepatitis C, and a low CD4 nadir predispose to immunological nonresponse, previous therapeutic failure, duration of antiretroviral therapy, advanced stage of disease, low adherence to HAART, and previous treatment interruption [12-15]. The roles of T-cell subsets, thymic function, and cytokines have been investigated. The increased T-cell activation has been associated with a lack of effective immunologic response. An important predictor of $\mathrm{CD} 4+\mathrm{T}$ cell reconstitution is the level of immune activation that contributes to $\mathrm{CD} 4+\mathrm{T}$ cell death via apoptosis, the level of CD38 expression on different T-cell 
subsets may influence the ability to reconstitute CD4+ T cells under successful highly active antiretroviral therapy [16].

The underlying mechanism is fundamentally due to increased or reduced production of CD4+ T lymphocytes. Decreased hematopoietic function and decreased thymic function output are the main causes of reduced production. The mechanisms of increased destruction mainly include immune activation, increased apoptosis, and steady-state imbalance of T cell subsets. AIDS is a very complex syndrome. It is impossible to clarify its complex immunopathology and clinical problems by relying solely on CD4+ T lymphocytes and viral load. There must be other factors involved in the pathogenesis of immune non-response. More and more studies now tend to think that $\mathrm{CD} 4+\mathrm{T}$ cell reduction is associated with abnormal immune activation after viral infection. The immune activation of chronically infected people continues to affect lymphocyte proliferation and apoptosis: ectopic flora and virus storage persist. Lymphoid tissue damage and other factors interact with immune activation, leading to damage of the mucosal barrier, affecting the distribution of CD4+ $\mathrm{T}$ cells in lymphoid tissues and blood, immune abnormal activation and immune non-response are causal and vicious cycles, making immune activation related research has become a top priority in the field of immune non-response. [17-18].

\section{Current Modern Medicine Treatment to Improve Immune Reconstitution}

So far, a range of supplementary treatment to HAART has been suggested to improve immune reconstitution. The only thoroughly investigated candidate has been IL-2 that unfortunately proved not to be beneficial for clinical outcome. Some antiviral drugs may be better than others in immunologic reconstitution. Protease inhibitors may have additional, independent positive effects on the immune system. On the other hand, there may be little rationale for using immunosuppressive agents such as cyclosporine or hydroxyurea in this subgroup of immunologic non responder patients, as these molecules may increase T-cell decline and/ or favor susceptibility to infections.[17]Several candidates seem promising including supplementary treatment with IL-7, possibly Cox-2 inhibitors. Furthermore, using Maraviroc as an integrated component of HAART does seem to result in higher CD4+ cell counts, but at present, the possibilities of improving the immune reconstitution in INR using supplementary treatment are limited. Some predictive factors can be avoided. Early diagnosis could be improved, reducing the risk for a low CD4 nadir, and coinfection with hepatitis $\mathrm{C}$ can be treated. Additional treatment has been investigated but results thus far have proved disappointing.

\section{The Role of Traditional Chinese Medicine to Improve Immune Reconstitution}

Traditional Chinese medicine has a unique role in the regulation of human immune system; Promoting immune reconstitution by Chinese medicine has become the focus in field of AIDS research.
TCM has been used in Chinese society for more than 5,000 years. It has been widely and successfully used to treat diseases from inflammation to cancer and retains an important role in the healthcare system of China today. Different from conventional western medicine system, TCM treating patients individually, the body is recognized and treated as a whole entity, and diseases are identified as conditions caused by internal imbalances. The role of doctors is to identify imbalances and then correct them; the body is then expected to be able to heal itself.

By reviewing published articles, we found that studies on traditional Chinese medicine to improve immune reconstitution usually from three aspects to assess immunomodulatory effects: 1) improve CD4 absolute values, naive CD4+ cell count and memory CD4+ cell count; 2) improve CD45RA and/or CD45R0; 3) decrease in T-cell immune activation. Immune reconstitution is an important aspect of management regimens of AIDS patients. TCM can benefit the Immune reconstitution and reduce the abnormal immune activation [19]. Randomly divided 361 AIDS patients with immune reconstitution deficiency after HAART into treatment group and control group, Patients of the treatment group have remarkably higher CD4+lymphocytes counts than the ones of the control group after 72-week treatment $(\mathrm{P}<0.05)$ [20].

A report of 6 months outcome on immune reconstitution of 228 cases of INR patients, patients were randomly divided into treatment group and control group, treatment group received combination therapy of Immune 1 Granule and HAART, control group was given placebo combined HAART, results showed that patients with CD4+ cell cells baseline level between 200 and 350 cells $/ \mu \mathrm{L}$, immune reconstitution rate of treatment group is significantly higher than the control group. Immune reconstitution rate here refers to CD 4 + cell cells increase $30 \%$ or 50 cells $/ \mu \mathrm{L}$ from baseline [21]. A randomized double-blind, placebo-controlled clinical trial was designed to observe the effect of Immune No. 2 granule on the INR patients after highly active antiretroviral therapy.223 patients were randomly divided into treatment group (116 cases, Immune No. 2 granule + HAART) and control group (117 cases, placebo + HAART) for 6 months. Results showed that after intervention for 6 months after treatment, the effective rate of the treatment group was significantly superior to the control group. CD4, CD45RA, CD45R0 count of the treatment group was significantly higher than that of the control group $(\mathrm{P}<0.05)$ [22]. Use of Tripterygium wilfordii Hook F (Tw HF) extract in HIV-infected patients was associated with a reduction in T-cell activation and improved CD4 recovery with an excellent safety profile. Eighteen cART-treated HIV-infected patients virally suppressed for over 12 months with suboptimal CD4 cell recovery were enrolled.

Tw HF extract was administered at a dosage of $10 \mathrm{mg}$ three times daily for 12 months. T-cell subsets and activation markers were evaluated at baseline and during follow-up TwHF extract was associated with a mean increase in CD4 cell count of 88 cells $/ \mu \mathrm{l}$ 
after one year of treatment. A significant increase in the mean rate of CD4 cell recovery ( 26 before vs 75 cells/ $\mu$ l/year after Tw HF use, $\mathrm{P}<0.001$ ) was observed. Analysis of 13 patients with activation profiles suggested that Tw HF extract was associated with a decrease in T-cell immune activation [23]. Regulating immune activation with TCM remains an important novel field of research. Immune activation is linked to and predictive of disease progression, and it has an additive or stronger prognostic value than does CD4+ cell count or viral load alone [24-26]. This is highlighted by the fact that a rare subgroup of HIV-infected patients, elite controllers, who do not progress, and sustain normal CD4+ cell counts and undetectable viral loads despite lack of treatment have a lower immune activation than normal progressors do [27]. Increased immune activation during primary HIV infection has been proposed to be predictive of CD4+ cell depletion and poor response to HAART [28].

A China phase III clinical muti-center placebo controlled doubleblind trial using Tangcao Tablets demonstrates significant increase in CD4 counts, CD4/CD8 and weight in herb group and significant decrease of viral load in placebo group [29]. A randomized doubleblind placebo-controlled clinical trial using Aifukang Capsule involving 198 patients showed significant effect in improving immune function and clinical symptoms such as fatigue, anorexia, headache, skin rash and insomnia [30]. Traditional Chinese medicine treatment emphasizes the overall adjustment of the body and adjusts the imbalance between yin and yang. Moderate adjustment of the state of inhibition and activation of the body's immune system is an important manifestation of the regulation of yin and yang in the body's immune regulation. Therefore, whether Chinese medicine can activate the abnormal activation state of immune reconstitution by HIV-infected patients with multi-target effect and two-way regulation is a breakthrough attempt. To study the mechanism of abnormal immune activation, it is a long way to clarify the mechanism of Chinese medicine intervention in immune non-response status.

\section{Acknowledgement}

The article Supported by National Major Science and Technology Specific Project of China (No. 2017zx10205501and 2017zx10205501001010); National Major Science and Technology Specific Project of China (No.2018ZX10101001005001) ; Independent Selection Project of China Academy of Chinese Medical Sciences(No.YZ-1662).National Clinical Research Base for Traditional Chinese Medicine( JDZX2015167).

\section{Conflict of Interest}

No conflict of interest.

\section{References}

1. Ye Ma, Zhihui Dou, Wei Guo, Yurong Mao, Fujie Zhang, et al. (2018) The Human Immunodeficiency Virus Care Continuum in China: 1985-2015, HIV Care Continuum in China. CID 66 (15): 833-839.

2. Kelley CF, Kitchen CM, Hunt PW, Rodriguez B, Hecht FM, et al. (2009) Incomplete peripheral CD4 + cell count restoration in HIV-infected patients receiving long-term antiretroviral treatment. Clinical Infectious Disease 48(6): 787-794.

3. Nakanjako D, Kiragga A, Ibrahim F, Castelnuovo B, Kamya MR, et al. (2008) Sub-optimal CD4 reconstitution despite viral suppression in an urban cohort on antiretroviral therapy (ART) in sub-Saharan Africa: frequency and clinical significance. AIDS Res Ther 5: 23.

4. Negredo E, Massanella M, Puig J, Gallego Escuredo JM, Villarroya J, et al. (2010) Nadir CD4 T cellcount as predictor and high CD4 T cell intrinsic apoptosis as final mechanism of poor CD4 T cell recovery in virologically suppressed HIVinfected patients: clinical implications. Clinical Infectious Disease50 (9): 1300-1308.

5. Marchetti G, Gazzola L, Trabattoni D, Bai F, Ancona G, et al. (2010) Skewed $\mathrm{T}$ cell maturation and function in HIV-infected patients failing CD4+ recovery upon long-term virologically suppressive HAART, AIDS, 24(10): 1455-1460.

6. Li T, Wu N, Dai Y, Han Y, Xie J, et al. (2011) Reduced thymic output is a major mechanism of immune reconstitution failure in HIV-infected patients after long-term antiretroviral therapy. Clinical Infectious Diseases 53 (9): 944-951.

7. Lewden C, Chene G, Morlat P, Raffi F, Dupon M, et al. (2007) HIV-infected adults with a CD4 cell count greater than 500 cells/mm3 on long-term combination antiretroviral therapy reach same mortality rates as the general population, Journal of Acquired Immune Deficiency Syndromes 46(1): 72-77.

8. (2008) Antiretroviral Therapy Cohort Collaboration, Life expectancy of individuals on combination antiretroviral therapy in high-income countries: a collaborative analysis of 14 cohort studies, Lancet, 372(9635): 293-299.

9. Engsig FN, Gerstoft J, Kronborg G, Carsten S Larsen, Gitte Pedersen, et al. (2010) Long-term mortality in HIV patients virally suppressed for more than three years with incomplete CD4 recovery: a cohort study, BMC Infectious Diseases 10: 318.

10. Bosch RJ, Wang R, Vaida F, Lederman MM, Albrecht MA (2006) Changes in the slope of the CD4 cell count increase after initiation of potent antiretroviral treatment. J Acquir Immune Defic Syndr 43: 433-435.

11. Lok JJ, Bosch RJ, Benson CA, Collier AC, Robbins GK, et al. (2010) Longterm increase in CD4+ T-cell counts during combination antiretroviral therapy for HIV-1 infection. AIDS 24: 1867-1876.

12. Moore RD, Keruly JC (2007) CD4+ cell count 6 years after commencement of highly active antiretroviral therapy in persons with sustained virologic suppression, Clinical Infectious Diseases 44(3): 441-446.

13. Amico RD, Yang Y, Mildvan D, Evans SR, Schnizlein Bick CT, et al. (2005) Lower CD4+ T lymphocyte nadirs may indicate limited immune reconstitution in HIV-1 infected individuals on potent antiretroviral therapy: analysis of immunophenotypic marker results of AACTG 5067. Journal of Clinical Immunology 25(2): 106-115.

14. Kaufmann GR, Furrer H, Ledergerber B, Perrin L, Opravil M, et al. (2005) Characteristics, determinants, and clinical relevance of CD4 $\mathrm{T}$ cell recovery to $<500$ cells $/ \mu \mathrm{L}$ in HIV type 1 -infected individuals receiving potent antiretroviral therapy, Clinical Infectious Diseases 41(3): 361372

15. Greub G, Ledergerber B, Battegay M, Grob P, Perrin L, et al. (2000) Clinical progression, survival, and immune recovery during antiretroviral therapy in patients with HIV-1 and hepatitis C virus coinfection: the swiss HIV cohort study. Lancet 356(9244): 1800-1805.

16. Benito JM, López M, Lozano S, Ballesteros C, Martinez P, et al. (2005) Differential upregulation of CD38 on different T-cell subsets may influence the ability to reconstitute CD4+ T cells under successful highly active antiretroviral therapy. J Acquir Immune Defic Syndr 38(4): 373381.

17. Aiuti F, Mezzaroma L (2006) Failure to reconstitute CD4+ T-cells despite suppression of HIV replication under HAART. AIDS Rev 8(2): 88-97. 
18. Beq S, Delfraissy JF, Theze J (2004) Interleukin-7 (IL-7): immune function, involvement in the pathogenesis of HIV infection and therapeutic potential. Eur Cytokine Netw 15(4): 279-289.

19. Yang Qiaoli, Zhen haofei, Liu Ying (2016) Warming Kidney for Immune Reconstruction in HIV/AIDS, Journal of Traditional Chinese Medicine 57(18): 1614-1615.

20. LIU Ying, WANG Jian, ZOU Wen (2017) Effect of Immune No. 2 Granule on CD4+ Counts of AIDS Patients with Immune Reconstitution Deficiency after HAART. J Journal of Traditional Chinese Medicine 58(1): 34-37.

21. Wu Xinfang, Wang Jie, Li Yong (2013) Intervention of Immune 1 combined HAART on immune function in patients with HIV/AIDS reconstruction, China Journal of Chinese Materia Medica 38(15): 2453-2457.

22. Wang Jie, Li Yong, Tang Yanli, Lin Hong sheng, Wu XF, et al. (2013) Effect of Immune No.2 on the Immune Reconstitution in patients with HIV/ AIDS after Highly Active Antiretroviral Treatment: A randomized Double blind placebo controlled clinical trial. Chinese Journal of Integrated Medicine 19(5): 340-346.

23. Li T, Xie J, Li Y, Routy JP, Li Y, et al. (2015) Tripterygium wilfordii Hook F extract in cART-treated HIV patients with poor immune response: a pilot study to assess its immunomodulatory effects and safety. HIV Clin Trials 16(2): 49-56

24. Hazenberg MD, Otto SA, Van Benthem BH, Roos MT, Coutinho RA, et al. (2003) Persistent immune activation in HIV-1 infection is associated with progression to AIDS, AIDS 17(13): 1881-1888.

ISSN: 2574-1241

DOI: 10.26717 /BJSTR.2019.22.003754

Ying LIU. Biomed J Sci \& Tech Res

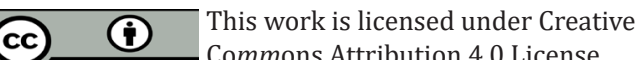

Submission Link: https://biomedres.us/submit-manuscript.php
25. Sousa AE, Carneiro J, Meier Schellersheim M, Grossman Z, Victorino RM (2002) CD4 T cell depletion is linked directly to immune activation in the pathogenesis of HIV-1 and HIV-2 but only indirectly to the viral load. Journal of Immunology169(6): 3400-3406.

26. Grossman Z, Meier Schellersheim M, Sousa AE, Victorino RM, Paul WE (2002) CD4+ T-cell depletion in HIV infection: are we closer to understanding the cause? Nature Medicine 8(4): 319-323.

27. Rachel E Owen, John W Heitman, Dale F Hirschkorn, Marion C Lanteri, Hope H Biswas, et al. (2010)HIV+ elite controllers have low HIV-specific T-cell activation yet maintain strong, polyfunctional T-cell responses, AIDS 24(8):1095-1105.

28. Gascon RL, Narváez AB, Zhang R, Kahn JO, Hecht FM ,et al. (2002) Increased HLA- 'DR expression on peripheral blood monocytes in subsets of subjects with primary HIV infection is associated with elevated CD4 T-cell apoptosis and CD4 T-cell depletion, Journal of Acquired Immune Deficiency Syndromes 30(2): 146-153.

29. Shao BP (2008) Clinical effect and safety report on HIV/AIDS patients with Tangcao tablets, in Proceedings of the 6th Conference on HIV/AIDS Treatment with TCM, China Association of Traditional Chinese Medicine, Anhui, China.

30. Wu H, Zhao M, Li XW, Yao C, Zhang AM (2012) Clinical observation of the efficacy and safety of Aifukang capsule in patients with HIV infections and AIDS. Chin J AIDS STD(China) 18: 434-437.

$\begin{array}{ll}\text { BIOMEDICAL } & \text { Assets of Publishing with us } \\ \text { RESEARCHES } & \text { - Global archiving of articles } \\ & \text { - Immediate, unrestricted online access } \\ & \text { - Rigorous Peer Review Process } \\ & \text { - Authors Retain Copyrights }\end{array}$

